\title{
First feed affects the expressions of microRNA and their targets in Atlantic cod
}

\author{
Teshome Tilahun Bizuayehu ${ }^{1}$, Tomasz Furmanek ${ }^{2}$, Ørjan Karlsen ${ }^{3,4}$, Terje van der Meeren ${ }^{3,4}$, \\ Rolf Brudvik Edvardsen ${ }^{2}$, Ivar Rønnestad ${ }^{5}$, Kristin Hamre ${ }^{6}$, Steinar D. Johansen ${ }^{1}$ and Igor Babiak ${ }^{1 *}$ \\ ${ }^{1}$ Faculty of Biosciences and Aquaculture, Nord University, Postbox 1490, 8049 Bodø, Norway \\ ${ }^{2}$ Institute of Marine Research, Postbox 1870, Nordnes, 5817 Bergen, Norway \\ ${ }^{3}$ Institute of Marine Research, Austevoll Research Station, 5392 Storebø, Norway \\ ${ }^{4}$ Hjort Centre for Marine Ecosystem Dynamics, Institute of Marine Research, Postbox 1870, Nordnes, 5817 Bergen, Norway \\ ${ }^{5}$ Department of Biology, University of Bergen, Postbox 7803, 5020 Bergen, Norway \\ ${ }^{6}$ National Institute of Nutrition and Seafood Research, Postbox 2029, Nordnes, 5817 Bergen, Norway \\ (Submitted 13 August 2015 - Final revision received 4 December 2015 - Accepted 4 January 2016 - First published online 9 February 2016)
}

\section{Abstract}

To our knowledge, there is no report on microRNA (miRNA) expression and their target analysis in relation to the type of the first feed and its effect on the further growth of fish. Atlantic cod (Gadus morhua) larvae have better growth and development performance when fed natural zooplankton as a start-feed, as compared with those fed typical aquaculture start-feeds. In our experiment, two groups of Atlantic cod larvae were fed reference feed (zooplankton, mostly copepods, filtered from a seawater pond) $v$. aquaculture feeds: enriched rotifers (Brachionus sp.) and later brine shrimp (Artemia salina). We examined the miRNA expressions of six defined developmental stages as determined and standardised by body length from first feeding for both diet groups. We found eight miRNA (miR-9, miR-19a, miR-130b, miR-146, miR-181a, miR-192, miR-206 and miR-11240) differentially expressed between the two feeding groups in at least one developmental stage. We verified the next-generation sequencing data using real-time RT-PCR. We found 397 putative targets (mRNA) to the differentially expressed miRNA; eighteen of these mRNA showed differential expression in at least one stage. The patterns of differentially expressed miRNA and their putative target mRNA were mostly inverse, but sometimes also concurrent. The predicted miRNA targets were involved in different pathways, including metabolic, phototransduction and signalling pathways. The results of this study provide new nutrigenomic information on the potential role of miRNA in mediating nutritional effects on growth during the start-feeding period in fish larvae.

\section{Key words: Artemia: Atlantic cod: Brachionus: Copepods: Live feed: microRNA: Nutrigenomics}

In oviparous fish, the transition from utilisation of yolk nutrients to exogenous feed requires considerable morphological, physiological and behavioural changes ${ }^{(1)}$. Survival and growth of a developing larva is determined by the development of functional organ systems and availability and quality of feed $^{(2-4)}$. Rotifers (mainly Brachionus sp.) followed by brine shrimp (Artemia salina) are the live prey most widely used for start-feeding of marine fish larvae in aquaculture. However, their nutritional value is suboptimal, resulting in inferior growth and performance compared with larvae fed natural zooplankton, such as in the case of Atlantic cod (Gadus morbua) larvae $e^{(3,4)}$

Studies on mammals and fish have shown that feed nutrient composition affects differential gene expression and activation of various metabolic pathways ${ }^{(5,6)}$, including modulation of microRNA (miRNA) ${ }^{(7,8)}$. miRNA are approximately twentytwo-nucleotide-long regulatory RNA present in animals and plants. They mainly pair with sites in the $3^{\prime}$ untranslated regions (3' UTR) of mRNA to mediate post-transcriptional repression ${ }^{(9)}$. The extent of sequence complementarity between miRNA and mRNA leads to control of protein production in the cell by blocking translation, degradation of the mRNA or both ${ }^{(10)}$. Many miRNA have pleiotropic effects and mediate a variety of physiological processes including differentiation, growth, development and energy metabolism ${ }^{(11,12)}$. In addition, miRNA expression is under less strict genetic control than that of mRNA $^{(13,14)}$, which indicates a possible regulation of miRNA by epigenetic mechanisms.

Metabolic regulation is a principal mechanism to control differentiation, proliferation and survival of cells ${ }^{(15)}$. Metabolic regulation depends on different intrinsic and extrinsic factors, including availability of nutrients. Lack of essential nutrients is among the major reasons of lower growth rate and deformities in Atlantic cod larvae fed rotifers compared with natural

Abbreviations: Calml4, calmodulin-like protein 4; c-myb, transcriptional factor myb; dph, day post-hatch; dusp5, dual-specificity phosphatase 5; IGF, insulinlike growth factor; igfals, insulin-like growth factor binding protein, acid-labile subunit; KEGG, Kyoto Encyclopedia of Genes and Genomes; miRNA, microRNA; UTR, untranslated regions.

* Corresponding author: I. Babiak, fax +47 75517 457, email igor.babiak@uin.no 
zooplankton diets ${ }^{(16,3)}$. miRNA act as essential metabolic rheostats at a cellular level ${ }^{(17)}$ and can boost mitochondrial ATP production $^{(18)}$. Therefore, these versatile regulatory molecules likely mediate nutritional effects on gene expression. However, limited information exists on miRNA control of metabolism and involvement in nutrient utilisation in teleost fish ${ }^{(19-21)}$. In juvenile rainbow trout (Oncorbynchus mykiss), expression of miR-122, a liver-specific miRNA, was altered with a change in dietary macronutrient ratio, particularly carbohydrate:protein and lipid:protein ratio ${ }^{(21)}$. Similarly, miR-143 expression increased after the commencement of exogenous feeding in rainbow trout alevins ${ }^{(20)}$. However, the only information on the link between miRNA regulation and the type and composition of larval feed is available from a study on rainbow trout alevins fed diets containing a high or very low protein:carbohydrate ratio, where the four studied miRNA - miR-29, miR-33, miR-107 and miR-143 - showed no differences in expression ${ }^{(22)}$. Previous experiments and observations have shown that the type of live feed has a profound effect on development as well as lifelong growth of Atlantic $\operatorname{cod}^{(3)}$.

This study provides a first insight into the nutrient-driven miRNA repertoire in fish larvae fed different types of live prey as a start-feed. Relationships between the differentially expressed miRNA and their putative target mRNA were also investigated. The results of this study provide some useful information on the role of miRNA in a metabolic network during fish larval development.

\section{Methods}

\section{Fish, experimental set-up and sampling}

The experiment was conducted at Austevoll Research Station of the Institute of Marine Research, Storebø, Norway (IMRAustevoll). Care and handling of fish used in this study were in accordance with the Norwegian Regulation on Animal Experimentation (The Norwegian Animal Protection Act no. 73 of 20 December 1974, Section 20-22, amended 19 June 2009). The specific experiment described in this study was approved by the local representative of the Norwegian Research Animal Committee (Forsøksdyrutvalget, www.fdu.no) with the acceptance reference FOTS id 5448.

A detailed description of experimental set-up, including live feed production and larval rearing, is described in the study by Karlsen et $a l .{ }^{(23)}$ and van der Meeren et al. ${ }^{(24)}$. Eggs were obtained from forty-five females and twenty-five males of Atlantic cod kept under natural photoperiod in a 7-m diameter indoor fibre-glass tank (average temperature: 7.9 (SD 0.3$)^{\circ} \mathrm{C}$ and salinity: $34.7(\mathrm{sD} 0.2) \mathrm{g} / \mathrm{l})$. The brood fish were 3 years old and in the range between 2 and $3 \mathrm{~kg}$, originating from broodstock maintained at IMR-Austevoll. They were fed Skretting Vitalis diet (Skretting) three times a week. Fish were spawning spontaneously every night during March and April 2012. Upon spawning, fertilised floating eggs were concentrated overnight in outlet collectors, disinfected with glutaraldehyde and incubated in six 70 litre flow-through egg incubators with gentle air bubbling around a centrally mounted outlet screen, at $6^{\circ} \mathrm{C}$ under continuous $24-\mathrm{h}$ roof light of $33 \mu \mathrm{W} / \mathrm{cm}^{2}$ intensity, until beyond hatching.
At 4-d post-hatch (dph), larvae were transferred from incubators to six black 400 litre start-feeding tanks. An equal number of larvae from each incubator (approximately 12500 larvae/incubator; 50000 in total) were stocked in each start-feeding tank (approximately 125 larvae/l). Before transfer, the start-feeding tanks were supplied with algal paste to produce turbidity (green water), beneficial for larval feeding ${ }^{(25)}$. Rotifers or zooplankton (various stages of copepods) were provided as a live feed separately to three tanks each, before larval transfer.

The tanks were divided into two treatment groups: the aquaculture feed group receiving the most common marine hatchery feed (rotifers, substituted later on with Artemia) and the reference group, which was fed zooplankton harvested from a seawater pond, predominately nauplii and copepodids of Acartia longiremis and Centropages hamatus ${ }^{(24)}$. In the first treatment, rotifers were enriched with LARVIVA Multigain (BioMar) and Sel-Plex ${ }^{\circledR}$ (Alltech) used as feed from 4 to $32 \mathrm{dph}$, with a gradual shift to Multigain-enriched Artemia from 32 to $35 \mathrm{dph}$, continuing with Artemia until $63 \mathrm{dph}$. Gradual switch to the formulated diet AgloNorse (Troms $\varnothing$ Fiskeindustri AS) took place from 58 to $63 \mathrm{dph}$. In the second treatment, larvae were fed zooplankton obtained from the seawater pond 'Svartatjern' (Austevoll, Norway). This comprised nauplii from 4 to $30 \mathrm{dph}$, copepodids from 18 to $44 \mathrm{dph}$ and gradual switch to AgloNorse from $37 \mathrm{dph}$. The amount of feed was adjusted daily by visual observation of the remaining prey in the tanks after feeding and evaluation of stomach fullness. Larvae were reared under $16 \mathrm{~h}: 8 \mathrm{~h}$ photoperiod and light intensity of $300-500 \mu \mathrm{W} / \mathrm{cm}^{2}$. The temperature was increased gradually from 8 to $11 \cdot 6^{\circ} \mathrm{C}$, from day 4 to day 11 post-hatch, respectively, more than $10 \mathrm{~d}$ before any observed differences in growth rates. Initial water flow was $1 \mathrm{l} / \mathrm{min}$; it increased to $6 \mathrm{l} / \mathrm{min}$ at switch to the formulated diet and to $10 \mathrm{l} / \mathrm{min}$ at experiment termination ${ }^{(23)}$.

Sampling dates in the two treatment groups were adjusted to larval size. Samples were collected when fish reached an average standard length of $4.5,5 \cdot 2,7 \cdot 0,9 \cdot 3,13.9$ and $25.1 \mathrm{~mm}$, which corresponded to 4, 11, 22, 29, 37 and $53 \mathrm{dph}$ in the reference feed group and to 4, 11, 22, 31, 54 and $71 \mathrm{dph}$ in the aquaculture feed group, respectively ${ }^{(23)}$. These six stages are named as stages $0-5$ throughout this paper. Whole larvae samples were snap-frozen in liquid $\mathrm{N}_{2}$ and stored at $-80^{\circ} \mathrm{C}$ until RNA extraction.

\section{RNA extraction and sequencing}

Total RNA was extracted using Trizol (Invitrogen) from at least six individuals from each rearing tank. Similarly, RNA was extracted from the live feeds (natural zooplankton, rotifers and Artemia nauplii). Quality of RNA was examined using a BioAnalyzer (Agilent Technologies). Samples with a 28S:18S RNA ratio $\geq 1.8$ and RNA integrity number $\geq 8$ were used for sequencing library preparation. Equal concentration of RNA was pooled from the replicate tanks. With the exception of stage 2 and $A$. nauplii samples, next-generation sequencing libraries were prepared following SOLiD Whole Transcriptome Analysis Kit protocol (Life Technologies) and sequenced using 5500xl SOLiD sequencer (Life Technologies) at the University of 
Nordland, Norway. Libraries from stage 2 and $A$. nauplii samples were prepared using NEXTflex Small RNA-Seq Kit v2 (Bioo Scientific) and sequenced using NextSeq 500 (Illumina) at the University of Oregon, USA. The use of two sequencing technologies did not affect the experimental set-up, because no temporal effects were investigated and all comparisons were performed for each stage separately. mRNA sequencing and data analysis were performed as described by Penglase et $a l^{(6)}$

\section{Characterisation of microRNA and differential expression analysis}

Adapter sequences and low-quality bases were trimmed using cutadapt $^{(26)}$. Sequences with high-quality score $(\geq 20)$ at least in the first half of the sequence were mapped to the Atlantic cod genome, gadMor $1^{(27)}$, using Bowtie $2^{(28)}$ with default setting. Conserved miRNA were identified using miRDeep $2^{(29)}$ by similarity searching against fish miRNA from miRBase $21^{(30)}$ and Atlantic cod miRNA ${ }^{(31)}$, whereas the search for conserved miRNA in rotifers, zooplankton and Artemia samples was performed against arthropods miRNA from miRBase $21^{(30)}$. The minimum threshold for miRNA count was set at 10 reads per million (rpm) at least in one of the samples. As RNA was extracted from the whole larvae, we also mapped small RNA sequences of Atlantic cod larvae to miRBase arthropods miRNA to check whether the miRNA that were expressed in the larvae were the same as those in the live feeds.

Differential expression of miRNA between the groups at six stages were assessed separately using NOISeq ${ }^{(32)}$. We used parameters as previously described ${ }^{(31)}$ with some differences. In this study, we considered differential expression between the groups, $q>0.95$ with threshold of $10000 \mathrm{rpm}$ counts difference and $0.8<q<0.95$ with threshold of $15000 \mathrm{rpm}$ counts difference. We used these stringent criteria to avoid false positives. This setting is reasonable, as NOISeq has a better correct calling for over-represented than under-represented genes ${ }^{(33)}$.

\section{Real-time RT-PCR}

To test the sequencing results, we performed real-time RT-PCR for the chosen miRNA: miR-19, miR-25, miR-203 and miR-204. Totally, six samples from each treatment (two pools from each tank) were used for real-time RT-PCR. Pooling was carried out by taking four random individuals for each pooled sample. Complementary DNA (cDNA) was synthesised using miRCURY LNA universal cDNA synthesis kit II (Exiqon). The reaction was performed on LightCycler 480 (Roche Applied Science) with SYBR $^{\circledR}$ Green chemistry using white ninety-six-well plates with thermal cycle conditions of $95^{\circ} \mathrm{C}$ for $10 \mathrm{~min}$, followed by forty-five cycles of $95^{\circ} \mathrm{C}$ for $10 \mathrm{~s}$ and $60^{\circ} \mathrm{C}$ for $1 \mathrm{~min}$. Cq values were collected using second derivative method. We used $5 \mathrm{~S}$ ribosomal RNA to normalise the expression levels of miR-19, miR-25, miR-203 and miR-204. The Shapiro-Wilk normality test was performed, and data not following normal distribution were log-transformed. The data fitting normality were analysed using Student's $t$ test, whereas the data that did not fit normality were analysed using non-parametric Mann-Whitney $U$ test.

\section{MicroRNA target prediction}

To predict miRNA targets, $3^{\prime}$ UTR of the Atlantic cod mRNA sequences were downloaded from NCBI database. We also obtained 3' UTR sequences from our RNA-Seq data (the Sequence Read Archive at NCBI, accession ID SRP056073). For this, we performed BLASTX search to peptide sequences of Atlantic cod Ensembl $79^{(27)}$ with $e$-value $10^{-4}$. We filtered sequences having similarity $>75 \%$ and considered only alignments covering stop codons. Next, we extracted sequences after the stop codon and annotated them as $3^{\prime}$ UTR. Potential miRNA-binding sites were identified using miRanda ${ }^{(34)}$ with strict seed matching, score $\geq 150$, and free energy threshold $\leq-83 \mathrm{~kJ} / \mathrm{mol}(\leq-20 \mathrm{kcal} / \mathrm{mol})$.

\section{Functional annotation of microRNA through ortholog assignment of their targets}

Targets of differentially expressed miRNA were annotated to Atlantic cod Ensembl 79 ${ }^{(27)}$ gene ontology (GO) terms. Similarly, miRNA targets were annotated with ortholog Kyoto Encyclopedia of Genes and Genomes (KEGG) genes ${ }^{(35)}$, using the best hit from a BLAST analysis against Danio rerio, Homo sapiens, Caenorhabditis elegans and Xenopus laevis with KEGG pathways annotation. Enrichment of terms was tested using g:Profiler ${ }^{(36)}$ for targets of differentially expressed miRNA at each stage. $P$ values were adjusted for multiple testing by Benjamini-Hochberg correction. $P<0.05$ was considered as statistically significant.

\section{Results}

\section{MicroRNA in Atlantic cod larvae and their live feed}

We obtained 112 million small RNA sequences from eleven Atlantic cod libraries, and on average $59 \%$ of them were mapped to Atlantic cod genome. miRNA constituted on average $26 \%$ of the total small RNA sequences. In addition, rotifers, zooplankton and Artemia libraries yielded more than ninety-six million small RNA sequences, which consisted of 0.44, 10 and 13\% miRNA, respectively (Table 1 , online Supplementary Table S1).

A number of conserved miRNA in both live feeds and Atlantic cod larvae were identified, but none of the arthropod-specific miRNA was considerably present in Atlantic cod larval small RNA libraries (online Supplementary Table S2). By considering $10 \mathrm{rpm}$ as a minimum miRNA count, we identified 348, 165, 95 and 70 mature miRNA in Atlantic cod larvae, rotifers, Artemia and zooplankton, respectively (online Supplementary Table S2). In spite of the fact that miRNA in rotifers had the highest diversity among the live feeds, approximately $90 \%$ of the total miRNA counts constituted of miR-87 and miR-125. The single most dominant miRNA in Artemia was bantam, whereas in the lagoon zooplankton sample the most abundant miRNA was miR-993, and the top five miRNA covered $70 \%$ of the total miRNA counts.

\section{Differential expression}

The effects of the type of live feed on larval development and performance, nutrient composition and the redox system have been reported in separate papers ${ }^{(23,6)}$. Generally, fish fed zooplankton (reference group) performed significantly better in 
Table 1. Overview of small RNA sequencing data*

(Numbers and percentages)

\begin{tabular}{|c|c|c|c|c|c|c|c|c|}
\hline & \multicolumn{2}{|c|}{ Atlantic cod larvae } & \multicolumn{2}{|c|}{ Rotifers } & \multicolumn{2}{|c|}{ Artemia } & \multicolumn{2}{|c|}{ Zooplankton } \\
\hline & $n$ & $\%$ & $n$ & $\%$ & $n$ & $\%$ & $n$ & $\%$ \\
\hline Total sequence counts & 112472052 & & 53683991 & & 32245495 & & 10756928 & \\
\hline Other ncRNA (\%) & 2484888 & $2 \cdot 2$ & & & & & & \\
\hline Mapped to miRNA with 0 mismatch (\%) & 25716773 & $22 \cdot 8$ & 77476 & 0.1 & 3636523 & $11 \cdot 3$ & 704716 & $6 \cdot 6$ \\
\hline Mapped to miRNA with 1 mismatch (\%) & 3042684 & $2 \cdot 7$ & 159742 & 0.3 & 551834 & 1.7 & 370285 & 3.4 \\
\hline Mapped to Atlantic cod genome (\%) & 65926532 & 58.6 & & & & & & \\
\hline
\end{tabular}

ncRNA, non-coding RNA; miRNA, microRNA.

* The total count represents clean adapter removed sequences. Sequences obtained from Atlantic cod larvae fed either aquaculture or reference live feeds (pooled five libraries each) were mapped to Atlantic cod miRNA, other ncRNA and genome; whereas sequences obtained from live feeds were mapped to arthropods miRNA (miRBase 21).

Table 2. MicroRNA expression in Atlantic cod larvae fed aquaculture (Brachionus sp. followed by Artemia sp.) or reference (zooplankton harvested from a lagoon, mostly copepods) diets $\dagger$

\begin{tabular}{|c|c|c|c|c|c|c|}
\hline & Stage 0 & Stage 1 & Stage 2 & Stage 3 & Stage 4 & Stage 5 \\
\hline miR-146-5p & 46 & & & & & \\
\hline Aquaculture & & 578 & $25435^{\star}$ & 1292 & 3751 & 1789 \\
\hline Reference & & 290 & 9066 & 942 & 1845 & 2157 \\
\hline miR-192-5p & 12251 & & & & & \\
\hline Aquaculture & & 33851 & $20995^{\star}$ & 39829 & 36713 & 44922 \\
\hline Reference & & 35704 & 9371 & 24951 & 19752 & 28052 \\
\hline miR-11240-5p & 2 & & & & & \\
\hline Aquaculture & & 268 & 58 & 225 & 86 & 38 \\
\hline Reference & & $10480^{\star}$ & $1920^{*}$ & $3567^{\star}$ & $1692^{*}$ & 7 \\
\hline$m i R-130 b$ & 42844 & & & & & \\
\hline Aquaculture & & 20366 & 9044 & 13149 & 10712 & 13553 \\
\hline Reference & & $32235^{\star}$ & 9205 & 16138 & 7929 & 16163 \\
\hline miR-9-5p & 10712 & & & & & \\
\hline Aquaculture & & 16532 & 62105 & $15398^{\star}$ & 11635 & 1404 \\
\hline Reference & & 9119 & $93151^{*}$ & 493 & 6742 & 3127 \\
\hline miR-206-3p & 89253 & & & & & \\
\hline Aquaculture & & 77214 & 40762 & 78442 & $126857^{*}$ & 182885 \\
\hline Reference & & 84254 & $56053^{*}$ & $152379^{*}$ & 66531 & 237350 \\
\hline miR-181a-5p & 12430 & & & & & \\
\hline Aquaculture & & 25094 & 47136 & 14525 & 9145 & 2630 \\
\hline Reference & & 19958 & $63383^{*}$ & 12021 & 15938 & 4264 \\
\hline miR-19a-3p & 21553 & & & & & \\
\hline Aquaculture & & 13404 & 14581 & 7132 & 23228 & 11720 \\
\hline Reference & & 12273 & 17683 & 9366 & $44727^{\star}$ & 10508 \\
\hline
\end{tabular}

Stage 0 , start of experiment.

`Significantly different (for details see the 'Methods' section).

$\dagger$ Read counts are normalised and presented as reads per million.

most measures - for example, they were significantly longer and heavier from $22 \mathrm{dph}$ onwards and had almost twice the growth rate $(4.5$ v. $2.4 \% / \mathrm{d})$ during this period ${ }^{(23)}$.

In the course of the experiment, eight miRNA showed significant differences in expression between the two feeding groups. At specific stages, differential expression was found for two miRNA at stage 1 , six miRNA at stage 2 , three miRNA at stage 3 , three miRNA at stage 4 and no miRNA was differentially expressed at stage 5 (Table 2). Expressions of some miRNA (miR-19a, miR-130b, miR-181a and miR-11240) were significantly higher in the reference feed group, some others (miR-146 and miR-192) had higher expression in the aquaculture feed group and few others (miR-9 and miR-206) showed mixed, developmental stage-dependent expression in the both feeding groups.

Verification of the NOISeq analysis with real-time RT-PCR analysis indicated no significant differences between the two feeding groups, except for miR-19d, miR-25 and miR-204 expressions at stage 1 (Fig. 1). The NOISeq analysis showed similar relations between miRNA expressions without false positives in the two feeding groups.

\section{Relation between differentially expressed microRNA and their potential targets}

We annotated the 3' UTR of 3698 genes, and 2770 of them had miRNA-binding sites for at least one of the Atlantic cod miRNA characterised in this study. Further analysis revealed that the eight differentially expressed miRNA could potentially target 397 mRNA (online Supplementary Table S3). Three mRNA were potential targets for three of these eight differentially expressed miRNA, and further forty-one mRNA were targets of two differentially expressed miRNA.

In all, eighteen of these 397 mRNA showed differential expression patterns across the study. They included transcriptional 

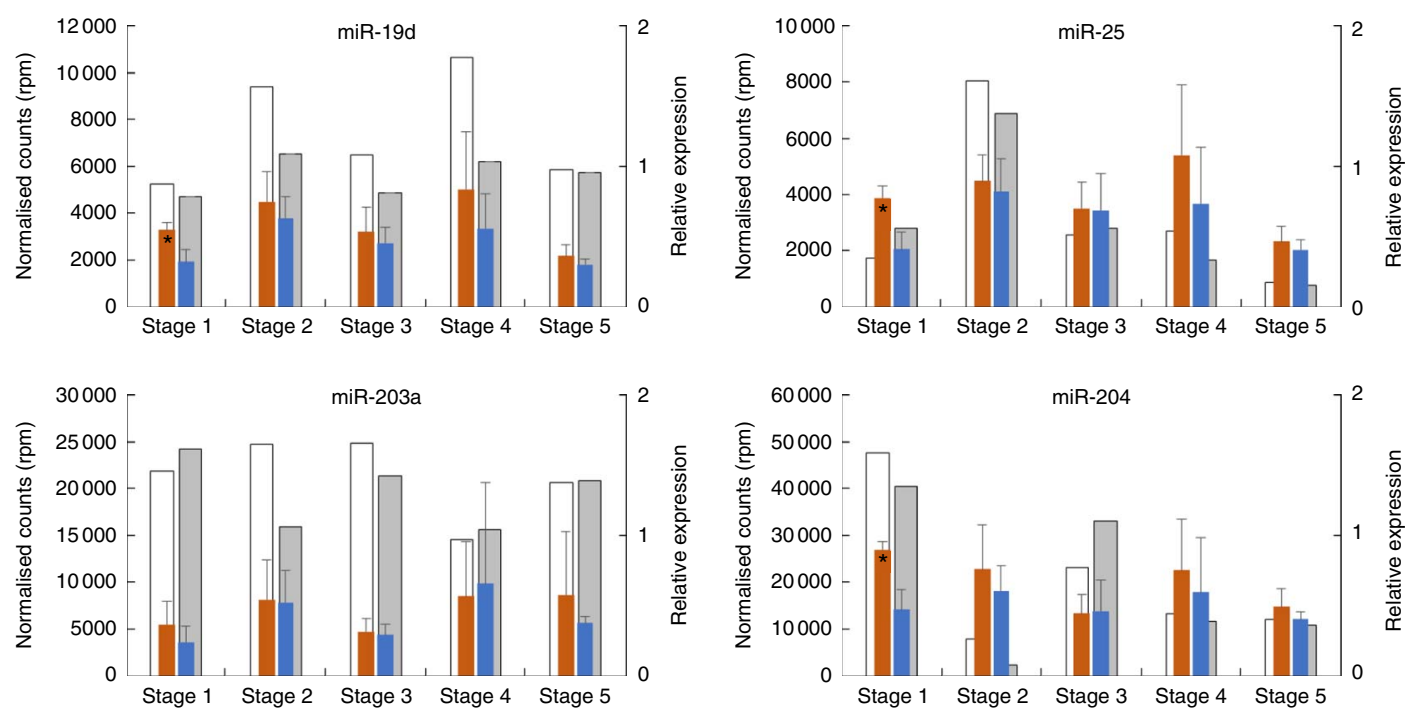

Fig. 1. Expression of microRNA obtained by next-generation sequencing (NGS) and real-time RT-quantitative PCR (RT-qPCR) from Atlantic cod larvae fed aquaculture (Brachionus sp. followed by Artemia sp.) or reference (zooplankton harvested from a seawater pond, mostly copepods) feeds and sampled at developmental stages $0-5$. For real-time RT-PCR, expression levels were normalised against $5 \mathrm{~S}$ ribosomal RNA. Values are means $(n 6)$, with standard deviation represented by error bars. * Significantly different between the two feeding groups within a stage: $P<0.05$. $\square$, Reference NGS; $\square$, aquaculture NGS; $\square$, reference RT-qPCR; $\square$, aquaculture RT-qPCR; rpm, reads per million.

factors, digestive enzymes, structural proteins, kinases and transporter transcripts (online Supplementary Table S4). The reciprocal relations of expression patterns of these mRNA and differentially expressed miRNA between the two feeding groups across the study (Fig. 2, online Supplementary Fig. S1) showed two general patterns: inverted, when higher miRNA expression in one of the feeding groups was accompanied by lower mRNA target expression in the same feeding group (Table 3), and concurrent, when both miRNA and its target mRNA expression were elevated within the same feeding group (online Supplementary Fig. S1). An inverse relation was observed, for example, at stage 2 , when a significant down-regulation of miR-9 and significant up-regulation of its targets insulin-like growth factor binding protein, acid-labile subunit (igfals) and chymotrypsin-like elastase family, member $2 A$ (cela $2 a$ ) was found in the aquaculture feed group as compared with the reference feed group. Similar inverse relation was found between dual-specificity phosphatase 5 (dusp5) and calmodulin-like protein 4 (calml4) and miR-181a (Fig. 2). Some other miRNA-mRNA pairs showed inverse relationships, but not significantly different between the two feeding groups in miRNA or mRNA expression at the same stage (Fig. 2). For instance, this was the case for miR-19a and collagen alpha-2(I) chain (col1a2) at stage 4, miR-206 and myb at stage 3, miR-9 and retinal $G$ proteincoupled receptor at stage 4 , as well as miR-9 and phosphorylase kinase gamma 1 (phkg1) at stage 4. Concurrent expression was observed for miR-206 and myb at stage 4 as well as miR-9 and cela $2 a$ at stage 3 (Fig. 2, online Supplementary Fig. S1).

\section{Enrichment for gene ontology terms and Kyoto Encyclopedia of Genes and Genomes pathways of differentially expressed microRNA targets}

In total, we identified 1291 GO terms and 146 KEGG pathways for the putative targets of miRNA identified in this study.
Differentially expressed miRNA targets were assigned in 324 molecular functions, seventy-three cellular components and 949 biological processes. These targets were involved in ninetyeight KEGG pathways. One KEGG term (KEGG:00670, one carbon pool by folate) was significantly enriched $(P=0.00669)$ at stages 3 and 4 . However, non-enriched targets were involved in various metabolic and signalling pathways and transportation networks (online Supplementary Table S3).

\section{Discussion}

In intensive aquaculture of most marine fish species including Atlantic cod, rotifers are used as a first feed followed by Artemia and later on by a formulated diet. These live feeds are deficient in certain nutrients and are usually enriched before being offered to the larva $\mathrm{e}^{(37)}$. Nevertheless, and despite large progress in enrichment protocols, replacement of these live feeds with natural zooplankton results in better performance parameters such as survival, growth, pigmentation, stress resistance and normal development ${ }^{(3,4,38)}$, which is attributed to superior nutritional composition of natural zooplankton ${ }^{(4,39)}$. In this study, we demonstrate that the nutritional effect of the first feed on larval and further juvenile performance of Atlantic cod may be related to a regulatory action of miRNA. However, global analysis of this regulation was limited by the resolution of the data and incomplete genomic resources. The low resolution resulted from technical limitations due to small larval size that prevented dissection of homogeneous tissues of relevance. Thus, RNA analysis was performed on pooled samples of larvae, which compromised the precision of transcriptome information. Lack of complete sequences of Atlantic cod 3' UTR could have also affected our enrichment analysis. The $3^{\prime}$ UTR sequences derived in this study represented only $17 \%$ of the Atlantic cod genes ${ }^{(40)}$. The analysis of teleost-specific miRNA 

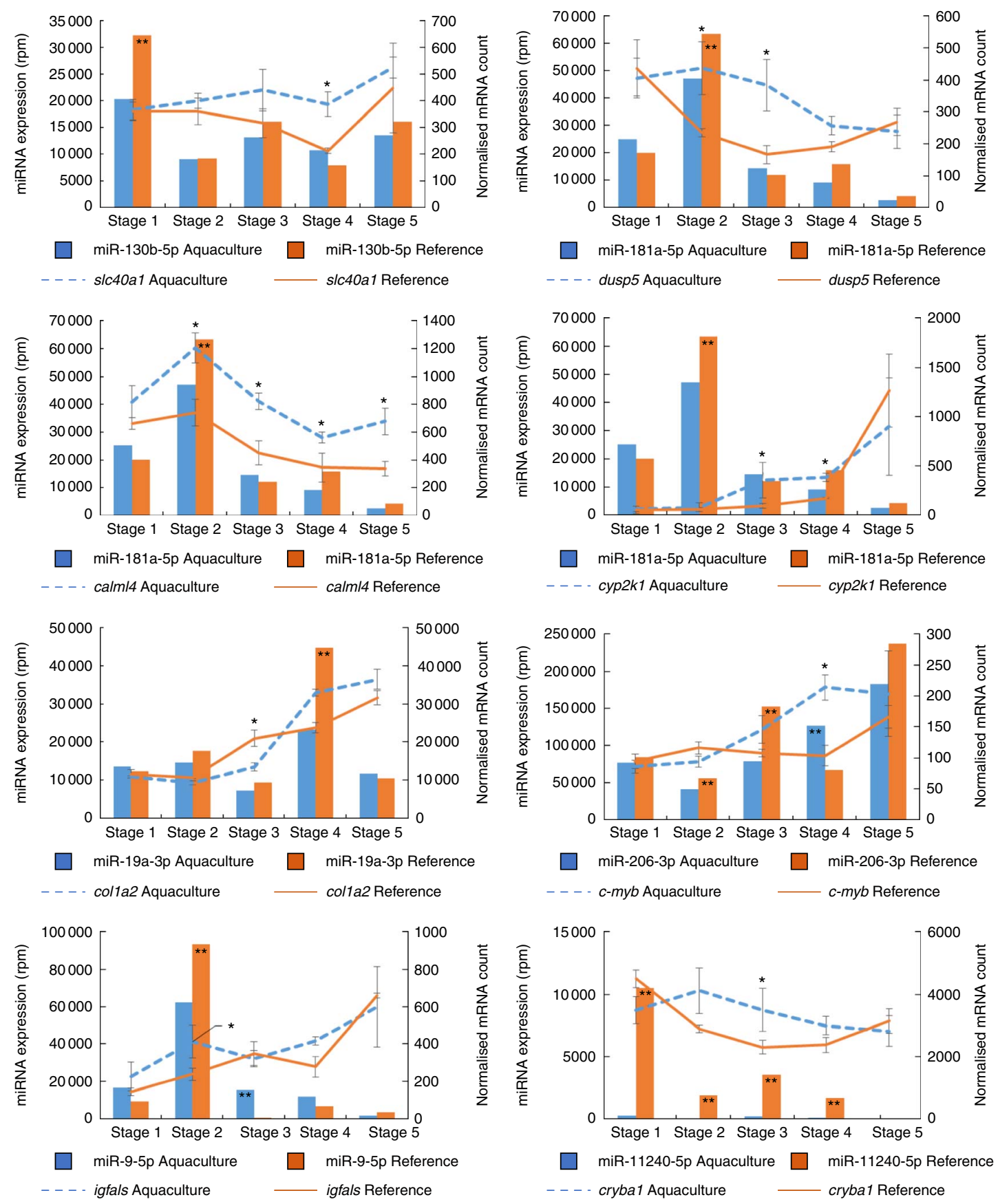

Fig. 2. Expression of selected microRNA (miRNA) and their putative mRNA targets in Atlantic cod fed aquacultured live feed $v$. reference natural zooplankton live feed. At stage 5 , both feeding groups were fed formulated diet. miRNA expression levels are given in reads per million (rpm), whereas mRNA reads are normalised by the total number of mapped sequences. Samples were taken at five larval stages and one juvenile stage. Values are means, with standard deviation represented by error bars. ${ }^{*}$ ** Significantly different expressions of mRNA and miRNA, respectively, between the two feeding groups within a stage ( $q>0.8$; for details see the 'Methods' section). slc40a1, Solute carrier family 40 (Fe-regulated transporter), member 1; dusp5, dual-specificity phosphatase 5; calm/4, calmodulin-like protein 4; cyp2k1, cytochrome P450 2K1; col1a2, collagen alpha-2(I) chain; igfals, insulin-like growth factor binding protein acid-labile subunit, c-myb, transcriptional factor myb; cryba1, crystallin, beta $A 1$.

generated 155.32 targets/miRNA on average ${ }^{(41)}$, whereas in this study the average number of targets for differentially expressed miRNA was 53.25 with a minimum of 12 and a maximum of 112/miRNA (online Supplementary Table S3). Aside from the incomplete set of $3^{\prime}$ UTR used, low number of significantly enriched pathways could result from diverse roles of differentially expressed miRNA in various biological pathways and/or functional redundancy of miRNA. The majority of miRNA acts in constellation and reduces their target level slightly, which has been shown in their noise reduction and in conferring precise protein level in the cell ${ }^{(42)}$.

In mammals, exogenous (dietary intake) miRNA are implicated in targeting low-density lipoprotein receptor adapter protein $1^{(43)}$. However, this regulation was found for miRNA 
Table 3. Inverse expressions of microRNA (miRNA) and their putative mRNA targets in Atlantic cod*

\begin{tabular}{|c|c|c|c|c|c|c|c|c|}
\hline \multirow[b]{2}{*}{ Inverse expression } & \multicolumn{2}{|c|}{ Stage 1} & \multicolumn{2}{|c|}{ Stage 2} & \multicolumn{2}{|c|}{ Stage 3} & \multicolumn{2}{|c|}{ Stage 4} \\
\hline & miRNA & Target & miRNA & Target & miRNA & Target & miRNA & Target \\
\hline miRNA up-target down & miR-130b & slc40a1 & $\begin{array}{l}\text { miR-9 } \\
\text { miR-181a }\end{array}$ & $\begin{array}{l}\text { cela2a, igfals } \\
\text { dusp5, calm/4 }\end{array}$ & miR-206 & $m y b$ & $\operatorname{miR}-19 a$ & col1a2 \\
\hline miRNA down-target up & & & $\operatorname{miR}-146$ & $m k n k 1$ & $\operatorname{miR}-9$ & lgfals, rgr, phkg1 & & \\
\hline
\end{tabular}

slc40a1, solute carrier family 40 (Fe-regulated transporter), member 1; cela2a, chymotrypsin-like elastase family, member 2A; igfals, insulin-like growth factor binding protein, acidlabile subunit, myb, transcriptional factor myb; col1a2, collagen alpha-2(I) chain; dusp5, dual-specificity phosphatase 5; calm/4, calmodulin-like protein 4; mknk1, MAP kinase interacting serine/threonine kinase 1; rgr, retinal G protein-coupled receptor, phkg1, phosphorylase kinase, gamma 1.

* Expression in reference feed group compared with aquaculture feed group is shown. Only miRNA and their putative targets each showing significant differential expression between the two feeding groups at different stages are shown.

originating from rice (Oryza sativa) as a diet. Plant miRNA have 2'-O-methylation at their $3^{\prime}$ ends, which protects them from exonucleolytic digestion ${ }^{(44)}$. Although extraction of RNA was performed using the whole organisms, we have not found considerable presence of live feed-specific (exogenous) miRNA. Although such effects cannot be ruled out, our data suggest that the feed components rather trigger endogenous mechanisms of gene expression in response to nutritional stimuli.

Several environmental factors such as temperature, photoperiod or feed have long-term effects on gene expression ${ }^{(45)}$. Recently, we demonstrated a long-lasting effect of embryonic thermal history on further miRNA expression in Atlantic cod, which was likely driven through an epigenetic mechanism ${ }^{(31)}$. Although we cannot rule out the effects of heterochrony or allometry on miRNA expression, as the two feeding groups elicited size difference as early as stage $2^{(23)}$, the epigenetic modulation of miRNA expression triggered by live feed components could contribute to ultimate phenotypic differences. However, we have not found a long-lasting effect of the first feed on miRNA expression in stage 5 , where both feeding groups were fed the same formulated diet.

miRNA may regulate $\mathrm{Fe}$ homoeostasis. In this study, we found a significantly higher expression of miR-130b in the reference feed group (mostly copepod nauplii) at stage 1 as compared with the rotifer-fed group (Table 2). Ferroportin or solute carrier family 40 (Fe-regulated transporter), member 1 is one of the predicted targets of miR-130b. It encodes a cell membrane protein that functions as an Fe exporter ${ }^{(46)}$. Dietary Fe absorbed by enterocytes can be stored in ferritin or exported by ferroportin, which is located at the basolateral surface of the enterocyte. In mammals, hepcidin, a hormone synthesised and secreted by hepatocytes, is the major regulator of Fe transport by inducing the endocytosis and proteolysis of ferroportin ${ }^{(47)}$. In several teleosts including Atlantic cod, hepcidin was identified and characterised as an antimicrobial peptide ${ }^{(48,49)}$. Although there is no defined dietary requirement for minerals, previous results suggest that rotifers contain sufficient amount of $\mathrm{Fe}$, which is ten times lower than that in natural zooplankton $^{(3,39)}$. Taken together, elevated expression of miR-130b in the reference feed group can be associated with translational repression of ferroportin. Thus, miR-130b may have a role in an additional or alternative mechanism for regulation of $\mathrm{Fe}$ homoeostasis.
The relation between miRNA and their putative targets could be through direct repressive interaction or indirect feedback or feed-forward mechanisms ${ }^{(50)}$. Although miRNA mainly suppress their targets, their roles in general gene regulatory networks need not to be simply suppressive. They could have gene regulatory network-dependent functions through miRNAtarget interactions. Hence, the identified miRNA-mRNA interactions in this study could be indirect. Whole larvae transcriptome sequencing and absence of a protein expression data set could confine our predicted functional analysis, as heterogeneity in tissues can complicate the analysis and not all miRNA-mRNA interactions confer mRNA degradation. In addition, miRNA and their targets have to co-localise to exert biological effects. For example, IGFALS is an insulin-like growth factor I (IGF-I) binding partner responsible for increasing the half-life of circulating IGF-I and for preventing possible non-specific metabolic effect of $\operatorname{IGF}^{(51)}$. Reduction of IGFALS reduces circulating IGF-I and, in consequence, growth. IGF-I has been implicated in somatic growth of Atlantic $\operatorname{cod}^{(52)}$. In this study, the expression of igfals mRNA had an inverse relationship with miR-9 in the reference feed group (Fig. 2), which showed faster growth ${ }^{(23)}$. miR-9 is mainly expressed in Atlantic cod brain ${ }^{(31)}$; however, it has been shown to be expressed in human blood serum ${ }^{(53)}$. Although IGFALS is produced in the liver, growth hormone $(\mathrm{GH})$ from the pituitary controls its production. miR-9 can be one of the rheostat of igfals in the GH/IGF axis. Therefore, the role of miR-9 in the accelerated growth of larvae in the reference feed group at stage 2 is either indirect or it does not exist in vivo.

miR-9 also targets phkg1 that activates glycogen phosphorylase, which further catalyses the rate-limiting step in glycogen breakdown. Larvae do not store a lot of glycogen ${ }^{(54)}$, but the reduction in miR-9 expression as development progresses towards the metamorphosis may suggest activation of the glycogenolysis pathway.

We found two targets with inverse expression patterns to that of miR-181a (Table 3, Fig. 2, online Supplementary Fig. S1). Among them, dusp5 is a negative regulator of p38 and stress-activated protein kinases/Jun amino-terminal kinases $(\mathrm{SAPK} / \mathrm{JNK})^{(55)}$, which are involved in signal transduction pathways with diverse biological functions ${ }^{(56)}$. In mammals, miR-181a is implicated in basal activation of T-cell receptor signalling molecules by repressing multiple negative regulators including DUSP5 ${ }^{(57)}$. In addition, mice carrying deletion of 
some of miR-181 clusters had impaired growth ${ }^{(17)}$. The significantly higher expression of miR-181a associated with significantly lower expression of dusp5 in the reference feed group as compared with rotifer group (online Supplementary Fig. S1) at stage 2 could result in better growth in the former group through better promotion of cellular differentiation and proliferation by suppressing the repressive action of dusp5. Calml4, one of multiple $\mathrm{Ca}^{2+}$-binding proteins involved in signal transduction, was another miR-181a target differentially expressed in this study. Calml4 is evolutionary conserved, expressed in all eukaryotic cells and involved in signalling pathways of diverse biological processes such as growth, proliferation, metabolism and transport ${ }^{(58)}$. Maternal transcripts of calml4 have been identified in unfertilised eggs of Atlantic $\operatorname{cod}^{(59)}$. Hamre et $a l .{ }^{(60)}$ reported higher content of Ca in rotifers as compared with zooplankton. Therefore, significantly higher expression of calml4 in the aquaculture feed group, as compared with the reference feed group (online Supplementary Fig. S1), could reflect the Ca content in a diet. The elevation of miR-181a expression in the reference feed group at stage 2 may indicate active involvement of miRNA-mediated suppression in the regulation of calml4 expression. However, it is unknown whether calml4, through $\mathrm{Ca}^{2+}$-dependent signalling pathway, has a role in the observed differences in larval performance between the two feeding regimens.

Col1a2 transcript, a component of extracellular matrix secreted by osteoblasts, is a target of miR-19a (Fig. 2). A significant enrichment in col1a2 has been reported in zebrafish $^{(61)}$ and sea bass (Dicentrarchus labrax) ${ }^{(62)}$ during larval development. In this study, both feeding groups showed reciprocal expressions of miR-19a and col1a2 at stage 4. In Atlantic cod, ossification occurs in larvae of size $11-20 \mathrm{~mm}^{(63,64)}$, which corresponds to stage 4 in this study. miR-19a is downregulated in osteoblast-like cells under peptide- 15 stimulation to promote bone formation ${ }^{(65)}$. Although the expression of miR-19a is higher in the reference feed group as compared with the aquaculture feed group at stage 4, its role in Atlantic cod bone formation and its mechanistic action is unknown yet.

miR-206 promotes cell differentiation by regulating transcriptional factor genes that are important in skeletal muscle growth. We predicted that miR-206 targeted a transcriptional factor $m y b(c-m y b)$. $C-m y b$ has been implicated in the proliferation of myoblasts by maintaining their undifferentiated states through inhibition of myogenic differentiation 1 $(m y o D)^{(66)}$. Myogenic regulatory factors (MGF) regulate the transcription of muscle-specific miRNA - for example, myogenic factor 5 (Myf5) and $M y o D$ regulate miR-206 transcription $^{(67)}$. At the same time, miR-206 forms a feedback regulatory loop with $\mathrm{MGF}^{(68)}$. Inhibition of miR-206 using antagomiR resulted in a substantially faster growth in Nile tilapia (Oreochromis niloticus) $^{(69)}$. Furthermore, ectopic expression of myoD increased expression of muscle-specific miRNA and induced muscle cell differentiation ${ }^{(70)}$. In Atlantic cod larvae, the expression of $m y o D 1$ increased and pax 7 decreased after $20 \mathrm{dph}^{(71)}$. The third phase of myogenesis (mosaic hyperplasia), which normally starts by the end of the larval period ${ }^{(72)}$, contains myogenic precursor cells that give rise to new myotubes ${ }^{(72)}$. In this study, the inverse relationship between
miR-206 and $c-m y b$ during metamorphosis in the reference feed group could reflect regulation of the $c-m y b$ by miRNA to accommodate the ongoing changes in skeletal muscle. This may suggest that the balance between proliferation and differentiation is important for fast larval growth. Therefore, suppression of $c-m y b$ by miR-206 could promote the transcription of $m y o D$, and thereby differentiation of myogenic precursor cells to myotubes, which may imply faster growth.

In this study, we identified differentially expressed miRNA that are known to be involved in different biological pathways. However, our enrichment analysis yielded only one significantly enriched pathway (one carbon pool by folate pathway), which had three miR-206 targets. Moreover, these targets were not differentially expressed between the two groups.

\section{Conclusion}

We demonstrate that the effect of the type of start-feed on larval development and growth likely implicates miRNA-mediated regulation. Different types of live feed resulted in differential expression of some miRNA and their putative targets throughout the feeding period. The effects were sometimes extended beyond the first feeding phase. As aquaculture live feeds (rotifers and Artemia) are nutritionally inferior to the reference feed (copepods), a specific enrichment of live feeds and/or feed modifications or supplementation to alter miRNA levels in larvae may offer a strategy to improve larviculture.

\section{Acknowledgements}

The study was financed by The Research Council of Norway, projects CODE (no. 199482) and FishmiR (no. 213825). The Research Council of Norway had no role in the design, analysis or writing of this article.

T. T. B. performed SOLiD Sequencing, real-time RT-PCR, analyses and wrote the first draft of the manuscript; T. F. and R. B. E. performed RNA-Seq and contributed to 3' UTR identification; $\varnothing$. K. and T. v. d. M. performed the experiment and sampling; I. R. and K. H. conceived and coordinated the experiment; S. D. J. and I. B. designed and coordinated the study. All the authors contributed to the writing of the manuscript.

The authors declare that there are no conflicts of interest.

\section{Supplementary material}

For supplementary material/s referred to in this article, please visit http://dx.doi.org/doi:10.1017/S0007114516000155

\section{References}

1. May RC (1974) Larval mortality in marine fishes and the critical period concept. In The Early Life History of Fish, pp. 3-19 [JS Blaxter, editor]. Berlin: Springer Berlin Heidelberg.

2. Sifa L \& Mathias JA (1987) The critical period of high mortality of larvae fish - a discussion based on current research. Chin J Oceanol Limnol 5, 80-96.

3. Imsland AK, Foss A, Koedijk R, et al. (2006) Short- and longterm differences in growth, feed conversion efficiency and deformities in juvenile Atlantic cod (Gadus morbua) startfed on rotifers or zooplankton. Aquacult Res 37, 1015-1027. 
4. Busch KET, Falk-Petersen I-B, Peruzzi S, et al. (2010) ) Natural zooplankton as larval feed in intensive rearing systems for juvenile production of Atlantic cod (Gadus morbua L.). Aquacult Res 41, 1727-1740.

5. Tacchi L, Secombes C, Bickerdike R, et al. (2012) Transcriptomic and physiological responses to fishmeal substitution with plant proteins in formulated feed in farmed Atlantic salmon (Salmo salar). BMC Genomics 13, 363.

6. Penglase S, Edvardsen RB, Furmanek T, et al. (2015) Diet affects the redox system in developing Atlantic cod (Gadus morhua) larvae. Redox Biol 5, 308-318.

7. Parra P, Serra F \& Palou A (2010) Expression of adipose microRNAs is sensitive to dietary conjugated linoleic acid treatment in mice. PLOS ONE 5, e13005.

8. Beckett EL, Yates Z, Veysey M, et al. (2014) The role of vitamins and minerals in modulating the expression of microRNA. Nutr Res Rev 27, 94-106.

9. Bartel D (2009) MicroRNAs: target recognition and regulatory functions. Cell 136, 215-233.

10. Djuranovic S, Nahvi A \& Green R (2012) miRNA-mediated gene silencing by translational repression followed by mRNA deadenylation and decay. Science 336, 237-240.

11. Dumortier O, Hinault C \& Van Obberghen E (2013) MicroRNAs and metabolism crosstalk in energy homeostasis. Cell Metab 18, 312-324.

12. Bizuayehu TT \& Babiak I (2014) MicroRNA in teleost fish. Genome Biol Evol 6, 1911-1937.

13. Parts L, Hedman ÅK, Keildson S, et al. (2012) Extent, causes, and consequences of small RNA expression variation in human adipose tissue. PLoS Genet 8, e1002704.

14. Siddle KJ, Deschamps M, Tailleux L, et al. (2014) A genomic portrait of the genetic architecture and regulatory impact of microRNA expression in response to infection. Genome Res 24, 850-859.

15. Williams A, Henao-Mejia J, Harman CCD, et al. (2013) miR-181 and metabolic regulation in the immune system. Cold Spring Harb Symp Quant Biol 78, 223-230.

16. Hamre K (2006) Nutrition in cod (Gadus morbua) larvae and juveniles. ICES J Mar Sci 63, 267-274.

17. Henao-Mejia J, Williams A, Goff LA, et al. (2013) The microRNA miR-181 is a critical cellular metabolic rheostat essential for NKT cell ontogenesis and lymphocyte development and homeostasis. Immunity 38, 984-997.

18. Zhang X, Zuo X, Yang B, et al. (2014) MicroRNA directly enhances mitochondrial translation during muscle differentiation. Cell 158, 607-619.

19. Mennigen JA, Panserat S, Larquier M, et al. (2012) Postprandial regulation of hepatic microRNAs predicted to target the insulin pathway in rainbow trout. PLOS ONE 7, e38604.

20. Mennigen JA, Skiba-Cassy S \& Panserat S (2013) Ontogenetic expression of metabolic genes and microRNAs in rainbow trout alevins during the transition from the endogenous to the exogenous feeding period. $J$ Exp Biol 216, 1597-1608.

21. Mennigen JA, Plagnes-Juan E, Figueredo-Silva CA, et al. (2014) Acute endocrine and nutritional co-regulation of the hepatic omy-miRNA-122b and the lipogenic gene fas in rainbow trout, Oncorbynchus mykiss. Comp Biochem Physiol B Biochem Mol Biol 169, 16-24.

22. Geurden I, Mennigen J, Plagnes-Juan E, et al. (2014) High or low dietary carbohydrate:protein ratios during first-feeding affect glucose metabolism and intestinal microbiota in juvenile rainbow trout. J Exp Biol 217, 3396-3406.

23. Karlsen $\varnothing$, van der Meeren T, Rønnestad I, et al. (2015) Copepods enhance nutritional status, growth and development in Atlantic cod (Gadus morbua L.) larvae - can we identify the underlying factors? PeerJ 3, e902.
24. van der Meeren T, Karlsen $\varnothing$, Liebig PL, et al. (2014) Copepod production in a saltwater pond system: a reliable method for achievement of natural prey in start-feeding of marine fish larvae. Aquacult Eng 62, 17-27.

25. van der Meeren T, Mangor-Jensen A \& Pickova J (2007) The effect of green water and light intensity on survival, growth and lipid composition in Atlantic cod (Gadus morhua) during intensive larval rearing. Aquaculture 265, 206-217.

26. Martin M (2011) Cutadapt removes adapter sequences from high-throughput sequencing reads. EMBnetjournal 17, 10-12.

27. Flicek P, Amode MR, Barrell D, et al. (2014) Ensembl 2014. Nucleic Acids Res 42, D749-D755.

28. Langmead B, Trapnell C, Pop M, et al. (2009) Ultrafast and memory-efficient alignment of short DNA sequences to the human genome. Genome Biol 10, R25.

29. Friedländer MR, Mackowiak SD, Li N, et al. (2012) miRDeep2 accurately identifies known and hundreds of novel microRNA genes in seven animal clades. Nucleic Acids Res 40, 37-52.

30. Kozomara A \& Griffiths-Jones S (2014) miRBase: annotating high confidence microRNAs using deep sequencing data. Nucleic Acids Res 42, D68-D73.

31. Bizuayehu TT, Johansen SD, Puvanendran V, et al. (2015) Temperature during early development has long-term effects on microRNA expression in Atlantic cod. BMC Genomics 16, 305 .

32. Tarazona S, García-Alcalde F, Dopazo J, et al. (2011) Differential expression in RNA-Seq: a matter of depth. Genome Res 21, 2213-2223.

33. Zheng X \& Moriyama E (2013) Comparative studies of differential gene calling using RNA-Seq data. BMC Bioinformatics 14, S7.

34. John B, Enright AJ, Aravin A, et al. (2004) Human microRNA targets. PLoS Biol 2, e363.

35. Kanehisa M, Araki M, Goto S, et al. (2008) KEGG for linking genomes to life and the environment. Nucleic Acids Res 36, D480-D484.

36. Reimand J, Arak T \& Vilo J (2011) g:Profiler - a web server for functional interpretation of gene lists (2011 update). Nucleic Acids Res 39, W307-W315.

37. Hamre K, Yúfera M, Rønnestad I, et al. (2013) Fish larval nutrition and feed formulation: knowledge gaps and bottlenecks for advances in larval rearing. Rev Aquacult 5, S26-S58.

38. Conceição LEC, Yúfera M, Makridis P, et al. (2010) Live feeds for early stages of fish rearing. Aquacult Res 41, 613-640.

39. Mæhre HK, Hamre K \& Elvevoll EO (2013) Nutrient evaluation of rotifers and zooplankton: feed for marine fish larvae. Aquacult Nutr 19, 301-311.

40. Star B, Nederbragt AJ, Jentoft S, et al. (2011) The genome sequence of Atlantic cod reveals a unique immune system. Nature 477, 207-210.

41. Yang L, Irwin DM \& He S (2015) Genome-wide identification and characterization of teleost-specific microRNAs within zebrafish. Gene 561, 181-189.

42. Schmiedel JM, Klemm SL, Zheng Y, et al. (2015) Gene expression. MicroRNA control of protein expression noise. Science 348, 128-132.

43. Zhang L, Hou D, Chen X, et al. (2012) Exogenous plant MIR168a specifically targets mammalian LDLRAP1: evidence of cross-kingdom regulation by microRNA. Cell Res 22, 107-126.

44. Yu B, Yang Z, Li J, et al. (2005) Methylation as a crucial step in plant microRNA biogenesis. Science 307, 932-935.

45. Jonsson B \& Jonsson N (2014) Early environment influences later performance in fishes. J Fish Biol 85, 151-188.

46. Donovan A, Brownlie A, Zhou Y, et al. (2000) Positional cloning of zebrafish ferroportin1 identifies a conserved vertebrate iron exporter. Nature $\mathbf{4 0 3}, 776-781$. 
47. Ganz T (2013) Systemic iron homeostasis. Physiol Rev 93 1721-1741.

48. Douglas SE, Gallant JW, Liebscher RS, et al. (2003) Identification and expression analysis of hepcidin-like antimicrobial peptides in bony fish. Dev Comp Immunol 27, 589-601.

49. Solstad T, Larsen AN, Seppola M, et al. (2008) ) Identification, cloning and expression analysis of a hepcidin cDNA of the Atlantic cod (Gadus morbua L.). Fish Shellfish Immunol 25, 298-310.

50. Tsang J, Zhu J \& van Oudenaarden A (2007) MicroRNAmediated feedback and feedforward loops are recurrent network motifs in mammals. Mol Cell 26, 753-767.

51. Boisclair Y, Rhoads R, Ueki I, et al. (2001) The acid-labile subunit (ALS) of the $150 \mathrm{kDa}$ IGF-binding protein complex: an important but forgotten component of the circulating IGF system. J Endocrinol 170, 63-70.

52. Davie A, Porter MJR, Bromage NR, et al. (2007) The role of seasonally altering photoperiod in regulating physiology in Atlantic cod (Gadus morhua). Part II. Somatic growth. Can J Fish Aquat Sci 64, 98-112.

53. Kong L, Zhu J, Han W, et al. (2011) Significance of serum microRNAs in pre-diabetes and newly diagnosed type 2 diabetes: a clinical study. Acta Diabetol 48, 61-69.

54. Conceição LEC, Verreth JAJ, Verstegen MWA, et al. (1998) A preliminary model for dynamic simulation of growth in fish larvae: application to the African catfish (Clarias gariepinus) and turbot (Scophthalmus maximus). Aquaculture 163, 215-235.

55. Tanoue T, Moriguchi T \& Nishida E (1999) Molecular cloning and characterization of a novel dual specificity phosphatase, MKP-5. J Biol Chem 274, 19949-19956.

56. Roux PP \& Blenis J (2004) ERK and p38 MAPK-activated protein kinases: a family of protein kinases with diverse biological functions. Microbiol Mol Biol Rev 68, 320-344.

57. Li QJ, Chau J, Ebert PJR, et al. (2007) miR-181a is an intrinsic modulator of T cell sensitivity and selection. Cell 129, 147-161.

58. Chin D \& Means AR (2000) Calmodulin: a prototypical calcium sensor. Trends Cell Biol 10, 322-328.

59. Lanes C, Bizuayehu T, de Oliveira Fernandes J, et al. (2013) Transcriptome of Atlantic cod (Gadus morbua L.) early embryos from farmed and wild broodstocks. Mar Biotechnol (NY) 15, 677-694.

60. Hamre K, Srivastava A, Rønnestad I, et al. (2008) Several micronutrients in the rotifer Brachionus sp. may not fulfil the nutritional requirements of marine fish larvae. Aquacult Nutr 14, 51-60.

61. Kessels MY, Huitema LFA, Boeren S, et al. (2014) Proteomics analysis of the zebrafish skeletal extracellular matrix. PLOS ONE 9, e90568.

62. Darias MJ, Zambonino-Infante JL, Hugot K, et al. (2008) Gene expression patterns during the larval development of European sea bass (Dicentrarchus labrax) by microarray analysis. Mar Biotechnol (NY) 10, 416-428.

63. Pedersen T \& Falk-Petersen IB (1992) Morphological changes during metamorphosis in cod (Gadus morbua L.), with particular reference to the development of the stomach and pyloric caeca. J Fish Biol 41, 449-461.

64. Herbing IHV, Miyake T, Hall BK, et al. (1996) Ontogeny of feeding and respiration in larval Atlantic cod Gadus morbua (Teleostei, Gadiformes): I. Morphology. J Morphol 227, 15-35.

65. Palmieri A, Pezzetti F, Brunelli G, et al. (2008) Peptide-15 changes miRNA expression in osteoblast-like cells. Implant Dent 17, 100-108.

66. Kaspar P, Pajer P, Sedlak D, et al. (2005) c-Myb inhibits myogenic differentiation through repression of MyoD. Exp Cell Res 309, 419-428.

67. Sweetman D, Goljanek K, Rathjen T, et al. (2008) Specific requirements of MRFs for the expression of muscle specific microRNAs, miR-1, miR-206 and miR-133. Dev Biol 321, 491-499.

68. Chen JF, Tao Y, Li J, et al. (2010) microRNA-1 and microRNA-206 regulate skeletal muscle satellite cell proliferation and differentiation by repressing Pax7. J Cell Biol 190, 867-879.

69. Yan B, Zhu CD, Guo JT, et al. (2013) miR-206 regulates the growth of the teleost tilapia (Oreochromis niloticus) through the modulation of IGF-1 gene expression. J Exp Biol 216, 1265-1269.

70. Koutsoulidou A, Mastroyiannopoulos N, Furling D, et al. (2011) Expression of miR-1, miR-133a, miR-133b and miR-206 increases during development of human skeletal muscle. $B M C$ Dev Biol 11, 34.

71. Hamre K, Penglase SJ, Rasinger JD, et al. (2014) Ontogeny of redox regulation in Atlantic cod (Gadus morhua) larvae. Free Radic Biol Med 73, 337-348.

72. Johnston IA (2006) Environment and plasticity of myogenesis in teleost fish. $J$ Exp Biol 209, 2249-2264. 